\title{
Molecular states with two heavy quarks
}

\author{
Ning Li \\ Institut für Kernphysik and Jülich Center for Hadron Physics \\ Forschungszentrum Jülich, D-52425 Jülich, Germany \\ Department of Physics and State Key Laboratory of Nuclear Physics \\ and Technology, Peking University, Beijing 100871, China \\ n.li@fz-juelich.de \\ Zhi-Feng Sun* and Xing Liu ${ }^{\dagger}$ \\ School of Physical Science and Technology \\ Lanzhou University, Lanzhou 730000, China \\ Research Center for Hadron and CSR Physics, Lanzhou University and \\ Institute of Modern Physics of CAS, Lanzhou 730000, China \\ *sunzhifo9@lzu.edu.cn \\ †xiangliu@lzu.edu.cn \\ Shi-Lin Zhu \\ Department of Physics and State Key Laboratory \\ of Nuclear Physics and Technology, Peking University, Beijing 100871, China \\ zhusl@pku.edu.cn
}

Published 16 April 2014

\begin{abstract}
Within the one-boson-exchange (OBE) potential model, we perform a coupled-channel study of the possible deuteron-like molecular states with two heavy flavor quarks. In our study, we take into account the S-D mixing and the coupled-channel effect in the flavor space. Interestingly, we predict some molecular states which are suggested to be searched for in the further experiments.
\end{abstract}

Keywords: Exotic states; molecular states; coupled-channel effects; double charm.

PACS Numbers: 14.40.Rt, 14.40.Lb, 12.39.Hg, 12.39.Pn

\section{Introduction}

With more and more experimental observations, the exotic state has become a hot topic. The molecular state explanation to the reported charmonium/bottomium-like

This is an Open Access article published by World Scientific Publishing Company. It is distributed under the terms of the Creative Commons Attribution 3.0 (CC-BY) License. Further distribution of this work is permitted, provided the original work is properly cited. 
states $X, Y, Z$ becomes the most popular one since many charmonium/bottomiumlike states are near the threshold of the charmed/bottomed meson pair, i.e.,

$$
\begin{aligned}
& X(3872) \sim m_{D \bar{D}^{*}}, \quad Z_{b}(10610) \sim m_{B \bar{B}^{*}} \\
& Y(4140) \sim m_{D_{s}^{*} \bar{D}_{s}^{*}}, \quad Z_{b}(10650) \sim m_{B^{*} \bar{B}^{*}} .
\end{aligned}
$$

And, in the past decade there are abundant literatures on the study of the heavy flavor molecular states. ${ }^{1-19}$

The concept of molecular state with hidden charm was first proposed by Voloshin and Okun thirty years ago. They studied the interaction between the charmed and anti-charmed mesons. ${ }^{20}$ Later, De Rujula, Georgi and Glashow suggested the observed $\psi(4040)$ to be a $D^{*} \bar{D}^{*}$ molecule. ${ }^{21}$ Using the quark-pion interaction model, Törnqvist investigated the possible deuteron-like bound states with $B \bar{B}^{*}$ or $B^{*} \bar{B}^{*}$ component. ${ }^{22,23}$

Usually, the hadron configurations mainly include

- Meson: $q \bar{q}, Q \bar{q}(q \bar{Q}), Q \bar{Q}$.

- Baryon: $q q q, Q q q, Q Q q, \ldots$

- Exotic states: Molecular state, Hybrid, Glueball....

Here, $q$ and $Q$ denote the light $(u, d, s)$ and heavy $(c, b)$ quarks, respectively. Among the conventional baryon states, the baryons with double charm or double bottom is of the $Q Q q$ configuration. The SELEX Collaboration reported the first observation of a doubly charmed baryon $\Xi_{c c}^{+}$in its charged decay mode $\Xi_{c c}^{+} \rightarrow \Lambda_{c}^{+} K^{-} \pi^{+24}$ and confirmed it in another decay mode $\Xi_{c c}^{+} \rightarrow p D^{+} K^{-} \cdot{ }^{25}$ Later the BABAR Collaboration searched for $\Xi_{c c}^{+}$in the final states $\Lambda_{c}^{+} K^{-} \pi^{+}$and $\Xi_{c}^{0} \pi^{+}$, and $\Xi_{c c}^{++}$ in the final states $\Lambda_{c}^{+} K^{-} \pi^{+} \pi^{-}$and $\Xi_{c}^{0} \pi^{+} \pi^{+}$, However, they found no evidence for the production of the doubly charmed baryons. ${ }^{26}$ The Belle Collaboration reported no evidence for the doubly charmed baryons in the final state $\Lambda_{c}^{+} K^{-} \pi^{+}$, either. ${ }^{27}$ Thus, it will be necessary and meaningful to continue to search for such doubly charmed baryons experimentally.

Besides the doubly charmed or bottomed baryons, it is also very interesting to study other systems with two heavy flavor quarks. The heavy flavor molecular state with two charm quarks provides us another approach to investigate the hadronic states with double charm. For this kind of hadron, its typical configuration is $[c \bar{q}][c \bar{q}]$. To answer whether there exist such heavy flavor molecular states or not, in this paper we apply the one-boson-exchange (OBE) potential model to perform a dynamic calculation of their mass spectroscopy. This study provides us new insight into exploring the hadronic states with double charm. Besides, we also investigate the states with double bottom or with both one charm and one bottom.

Using the one-boson-exchange potential model, we investigate the possible $D^{(*)} D^{(*)}, \bar{B}^{(*)} \bar{B}^{(*)}$ and $D \bar{B}^{(*)}$ molecules. In our study, we consider the S-D mixing because of the tensor force arising from the pseudoscalar exchange or the vector meson exchange. Particularly, we consider the coupled-channel effects in the flavor space. 
We first derive the effective potential in the momentum space. Since the heavy mesons are not point-like particles, we introduce one monopole form factor,

$$
F(q)=\frac{\Lambda^{2}-m_{e x}^{2}}{\Lambda^{2}-q^{2}}
$$

at exchange vertex, and make fourier transformation

$$
V(r)=\frac{1}{(2 \pi)^{3}} \int d \mathbf{q}^{3} e^{-i \mathbf{q} \cdot \mathbf{r}} V(\mathbf{q}) F^{2}(\mathbf{q}) .
$$

We obtain the effective potentials in the coordinate space, which have the forms:

$$
\begin{aligned}
& \mathcal{V}^{p}=\mathcal{V}_{\text {spin-spin }}^{p}+\mathcal{V}_{\text {tensor }}^{p} \\
& \mathcal{V}^{v}=\mathcal{V}_{\text {center }}^{v}+\mathcal{V}_{\text {spin-spin }}^{v}+\mathcal{V}_{\text {tensor }}^{v} \\
& \mathcal{V}^{s}=\mathcal{V}_{\text {center }}^{s}+\mathcal{V}_{\text {orbit-spin }}^{s} .
\end{aligned}
$$

In the above, the superscripts $p, v, s$ denote the pseudoscalar, vector and scalar meson exchange, respectively, while $\mathcal{V}_{\text {center }}^{i}, \mathcal{V}_{\text {spin-spin }}^{i}$, and $\mathcal{V}_{\text {tensor }}^{i}$ mean the center potential, spin-spin interaction and tensor potential, respectively.

Solving the coupled-channel Shördinger equation with the effective potential in the coordinate space, one obtains the numerical results including the mass, the binding energy, the root-mean-square radius and the probability of the individual channel. In the study, only the cutoff is a free parameter. However, due to the lack of the relevant experimental data, one can not determine the cutoff exactly. Here, we choose the range of the cutoff $0.9 \mathrm{GeV}<$ cutoff $<2.5 \mathrm{GeV}$ which is extracted from the study of the deuteron within the same meson-exchange model.

\section{The Systems with Strangeness 0}

For the systems with non-strangeness, the long range pion-exchange is permitted. In order to make clear the specific role of the long range pion-exchange force in the formation of the bound states, we first present the results with the one-pionexchange (OPE) potential, and then give the results with the one-boson-exchange (OBE) potential.

\section{1. $D^{(*)} D^{(*)}$}

For the $D^{(*)} D^{(*)}$ system, we investigate six states in all: $D^{(*)} D^{(*)}\left[I\left(J^{p}\right)=\right.$ $\left.0\left(0^{+}\right), 0\left(1^{+}\right), 0\left(2^{+}\right), 1\left(0^{+}\right), 1\left(1^{+}\right), 1\left(2^{+}\right)\right]$. The state $D^{(*)} D^{(*)}\left[I\left(J^{P}\right)=0\left(0^{+}\right)\right]$is forbidden because the present boson system should satisfy the Boson-Einstein statistic. However, the state $D^{(*)} D^{(*)}\left[0\left(1^{+}\right)\right]$is very interesting. Using the OPE potential , one obtains a loosely bound state with a reasonable cutoff, around $1.05 \mathrm{GeV}$. The binding energy relative to the $D D^{*}$ threshold is $1.24 \mathrm{MeV}$ and the corresponding root-mean-square radius is $3.11 \mathrm{fm}$ which is comparable to the size of the deuteron (about $2.0 \mathrm{fm}$ ). The dominant channel is $\frac{1}{\sqrt{2}}\left[D D^{*}-D^{*} D\right]\left({ }^{3} S_{1}\right)$, with a probability $96.39 \%$. Using the OBE potential instead of the OPE potential, the binding becomes 


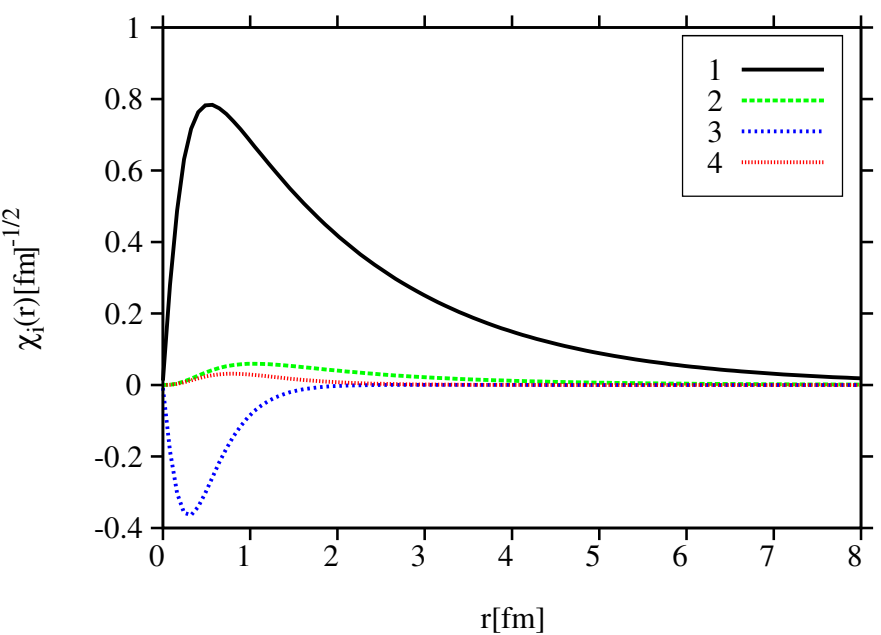

Fig. 1. (Color online). The wave function $(\mathrm{r} \psi(r))$ of the $D^{(*)} D^{(*)}$ system with strangeness 0 and $I\left(J^{P}\right)=0\left(1^{+}\right)$when the cutoff parameter is fixed to be $1.00 \mathrm{GeV}$.

tighter. Beside, we also find that the heavier rho and omega exchanges cancel each other significantly, which can be easily understood since for the isospin-zero system the isospin factor of $\rho$ is -3 while that of $\omega$ is 1 . To better understand this state, we also plot its radical wave function in Fig. 1, from which one can see clear that there is no node except for the origin. In other words, it is really a ground state.

We should mention that in the study of the $X(3872)$ one also obtained a bound $D \bar{D}^{*}$ state with quantum numbers $I\left(J^{P C}\right)=0\left(1^{++}\right)$using the OPE potential. ${ }^{28,29}$ One may be confused since there exists a G-parity rule between $D \bar{D}^{*}$ and $D D^{*}$, and the G-parity of the pion is odd. Actually, the iso-singlet $D \bar{D}^{*}$ system has two states with different $\mathrm{C}$-parities, one with even $\mathrm{C}$-parity $(C=+)$ and the other one with odd C-parity $C=-$ ). Our present $D D^{*}$ state relates to the $D \bar{D}^{*}$ state with odd C-parity but not with even C-parity via the G-parity rule.

We obtain no bound states of $D^{(*)} D^{(*)}\left[0\left(2^{+}\right)\right]$or $D^{(*)} D^{(*)}\left[1\left(0^{+}\right)\right]$for the cutoff less than $2.50 \mathrm{GeV}$ with either OPE potential or OBE potential. Thus, neither of the these two states are supported to be molecular candidates. We obtain no bound state of $D^{(*)} D^{(*)}\left[1\left(1^{+}\right)\right]$with the OPE potential for the cutoff being less than 2.50 $\mathrm{GeV}$, either. However, using the OBE potential, a bound state appears if the cutoff is fixed to be lager than $2.48 \mathrm{GeV}$. The result of the $I\left(J^{P}\right)=1\left(2^{+}\right)$state is similar to that of the $I\left(J^{P}\right)=1\left(1^{+}\right)$state.

\section{2. $\bar{B}^{(*)} \bar{B}^{(*)}$}

Due to the heavy quark flavor symmetry, $\bar{B}^{(*)} \bar{B}^{(*)}$ has many characteristics similar to those of $D^{(*)} D^{(*)}$. We investigate six states, $I\left(J^{P}\right)=$ $0\left(0^{+}\right), 0\left(1^{+}\right), 0\left(2^{+}\right), 1\left(0^{+}\right), 1\left(1^{+}\right)$and $1\left(2^{+}\right)$for $\bar{B}^{(*)} \bar{B}^{(*)}$, just as we do for $D^{(*)} D^{(*)}$. In the heavy quark limit, the potential of $\bar{B}^{(*)} \bar{B}^{(*)}$ is similar to that of 
$D^{(*)} D^{(*)}$ system. The main difference between the two systems is that the reduced mass of $\bar{B}^{(*)} \bar{B}^{(*)}$ is much larger than that of $D^{(*)} D^{(*)}$.

Among the three iso-scalar states, $I\left(J^{P}\right)=0\left(0^{+}\right), 0\left(1^{+}\right), 0\left(2^{+}\right)$, the one with $I\left(J^{P}\right)=0\left(0^{+}\right)$is forbidden because of the same reason as its charmed partner. Similar to the charmed case, one obtains a loosely bound state of $\bar{B}^{(*)} \bar{B}^{(*)}\left[0\left(1^{+}\right)\right]$with a reasonable cutoff using either OPE or OBE potential. Thus, it is supported to be a molecular candidate. Although one also obtains binding solutions of $\bar{B}^{(*)} \bar{B}^{(*)}\left[0\left(2^{+}\right)\right]$ using the OBE potential, one can not make a definite conclusion because of the strong dependence of the results on the cutoff.

For the three iso-vector states, one obtains a loosely bound state of $\bar{B}^{(*)} \bar{B}^{(*)}\left[I\left(J^{P}\right)=1\left(0^{+}\right)\right]$with the OPE potential for the cutoff being around 1.70 $\mathrm{GeV}$ or with the OBE potential for the cutoff being around $1.90 \mathrm{GeV}$. Actually, two pseudoscalar $\bar{B}$ mesons do not interact with each other via exchanging a pion. Therefore, the binding totally arises from the coupled-channel effect, just similar to the $\Lambda_{Q} \Lambda_{Q}$ case. $^{30,31}$ The state $\bar{B}^{(*)} \bar{B}^{(*)}\left[I\left(J^{P}\right)=1\left(1^{+}\right)\right]$is very interesting. One obtains a loosely bound state with OPE potential or OBE potential. Its binding energy is $0.83 \sim 11.80 \mathrm{MeV}$ with the OPE potential for the cutoff being $1.40 \sim 1.70$ $\mathrm{GeV}$ or $8.29 \mathrm{MeV}$ with the OBE potential for the cutoff being $1.70 \mathrm{GeV}$. Thus, we predict that $\bar{B}^{(*)} \bar{B}^{(*)}\left[I\left(J^{P}\right)=1\left(1^{+}\right)\right]$might be a molecule. We keep three channels, $\bar{B}^{*} \bar{B}^{*}\left({ }^{5} S_{2}\right), \bar{B}^{*} \bar{B}^{*}\left({ }^{1} D_{2}\right)$ and $\bar{B}^{*} \bar{B}^{*}\left({ }^{5} D_{2}\right)$, for the state $\bar{B}^{(*)} \bar{B}^{(*)}\left[I\left(J^{P}\right)=0\left(2^{+}\right)\right]$. Using the OPE potential, one obtains a loosely bound state with binding energy $2.25 \sim 13.35 \mathrm{MeV}$ for the cutoff being $1.80 \sim 2.10 \mathrm{GeV}$. Using the OBE potential, the binding energy is $7.88 \sim 28.01 \mathrm{MeV}$ for the cutoff being $1.70 \sim 1.76 \mathrm{GeV}$. Our results indicate that $1\left(2^{+}\right)$of $\bar{B}^{(*)} \bar{B}^{(*)}\left[1\left(2^{+}\right)\right]$might also be a molecular candidate.

\section{3. $D^{(*)} \bar{B}^{(*)}$}

Different from $D^{(*)} D^{(*)}$ or $\bar{B}^{(*)} \bar{B}^{(*)}, D^{(*)} \bar{B}^{(*)}$ has even more channels because $D^{(*)} \bar{B}^{(*)}$ does not need to meet the Boson-Einstein statistic. Using the OPE potential, we obtain a loosely bound state of $D^{(*)} \bar{B}^{(*)}\left[I\left(J^{P}\right)=0\left(0^{+}\right)\right]$for the cutoff being $1.08 \sim 1.20 \mathrm{GeV}$. When we add the contributions of the heavier eta, sigma, rho and omega exchanges, the binding energy changes by tens of $\mathrm{MeV}$. Our results suggest that $D^{(*)} \bar{B}^{(*)}\left[I\left(J^{P}\right)=0\left(0^{+}\right)\right]$might be a molecule candidate, but not a ideal one beause the results depend a little sensitively on the cutoff.

For the state $D^{(*)} \bar{B}^{(*)}\left[0\left(1^{+}\right)\right]$, the OPE potential is sufficient to form a bound state for the cutoff being lager than $2.05 \mathrm{GeV}$. Using the OBE potential, one obtains a loosely bound state of $D^{(*)} \bar{B}^{(*)}\left[0\left(1^{+}\right)\right]$state with an even more reasonable cutoff $1.65 \sim 1.80 \mathrm{GeV}$. Our present results suggest that $D^{(*)} \bar{B}^{(*)}\left[I\left(J^{P}\right)=0\left(1^{+}\right)\right]$might be a molecule candidate.

The state $D^{(*)} \bar{B}^{(*)}\left[I\left(J^{P}\right)=0\left(2^{+}\right)\right]$is particularly interesting. With the OPE potential, one obtains a loosely bound state with binding energy $0.80 \sim 3.83 \mathrm{MeV}$ for the cutoff being $2.10 \sim 2.30 \mathrm{GeV}$. Using the OBE potential, the cutoff decreases 
to $1.90 \sim 2.10 \mathrm{GeV}$ for a loosely bound state with binding energy $0.63 \sim 1.79 \mathrm{MeV}$. And, we predict $D^{(*)} \bar{B}^{(*)}\left[I\left(J^{P}\right)=0\left(2^{+}\right)\right]$to be a molecular candidate.

Using the OPE potential, none of the three iso-vector states: $D^{(*)} \bar{B}^{(*)}\left[1\left(0^{+}\right)\right.$, $1\left(1^{+}\right), 1\left(2^{+}\right)$] can form bound states with the cutoff less than $2.50 \mathrm{GeV}$. With the OBE potential, the results of the $J^{P}=0^{+}$state are similar to those of the $J^{P}=2^{+}$ state. Both of them form bound states with cutoff around $2.20 \mathrm{GeV}$. However, one obtains no bound state of $D^{(*)} \bar{B}^{(*)}\left[1\left(1^{+}\right)\right]$with cutoff less than $2.50 \mathrm{GeV}$. Thus, $D^{(*)} \bar{B}^{(*)}\left[1\left(1^{+}\right)\right]$is not supported to be a molecular candidate.

\section{The Systems with Strangeness 1}

For the systems with strangeness 1 , there is no pion-exchange, but the heavier eta, $K$ and $K^{*}$ exchanges are allowed.

\section{1. $\left(D^{(*)} D^{(*)}\right)_{s}$ and $\left(\bar{B}^{(*)} \bar{B}^{(*)}\right)_{s}$}

We obtain no bound state of $\left(D^{(*)} D^{(*)}\right)_{s}\left[J^{P}=0^{+}\right]$with the cutoff less than 2.50 $\mathrm{GeV}$. Differently, we obtain a loosely bound state of $\left(\bar{B}^{(*)} \bar{B}^{(*)}\right)_{s}\left[J^{P}=0^{+}\right]$with the cutoff being $1.82 \sim 1.88 \mathrm{GeV}$. Its binding energy is $0.56 \mathrm{GeV}$ and root-mean-square is $2.28 \mathrm{fm}$ which is comparable to that of the deuteron (about $2.0 \mathrm{fm}$ ). Thus, our results suggest that $\left(\bar{B}^{(*)} \bar{B}^{(*)}\right)_{s}\left[J^{P}=0^{+}\right]$might be a molecular candidate whereas $\left(D^{(*)} D^{(*)}\right)_{s}\left[J^{P}=0^{+}\right]$is not.

If we set the cutoff to be $1.44 \mathrm{GeV}$, the binding energy and root-meansquare radius of the charmed state $\left(D^{(*)} D^{(*)}\right)_{s}\left[J^{P}=1^{+}\right]$are $5.43 \mathrm{MeV}$ and 1.36 $\mathrm{fm}$, respectively. The closeness of the threshold of $D D_{s}^{*}$ to that of $D^{*} D_{s}$ makes the channels $D D_{s}^{*}\left({ }^{3} S_{1}\right)$ and $D^{*} D_{s}\left({ }^{3} S_{1}\right)$ provide the comparable and main contributions, $45.80 \%$ for $D D_{s}^{*}\left({ }^{3} S_{1}\right)$ and $51.31 \%$ for $D^{*} D_{s}\left({ }^{3} S_{1}\right)$. Our results indicate that $\left(D^{(*)} D^{(*)}\right)_{s}\left[J^{P}=1^{+}\right]$might be a molecule candidate. The results of $\left.\left(\bar{B}^{(*)} \bar{B}^{(*)}\right)_{s}\left[J^{P}=1^{+}\right]\right)$are similar to those of $\left(D^{(*)} D^{(*)}\right)_{s}\left[J^{P}=1^{+}\right]$, but with smaller cutoff $1.10 \sim 1.16 \mathrm{GeV}$ and weaker dependence on the cutoff. And, our result indicates that $\left.\left(\bar{B}^{(*)} \bar{B}^{(*)}\right)_{s}\left[J^{P}=1^{+}\right]\right)$seems to be an ideal molecular candidate.

The results of the $J^{P}=2^{+}$states are very similar to those of the $J^{P}=0^{+}$ states.

\section{2. $\left(D^{(*)} \bar{B}^{(*)}\right)_{s}$}

We obtain a bound state of $\left(D^{(*)} \bar{B}^{(*)}\right)_{s}\left[J^{P}=0^{+}\right]$with binding energy $6.72 \mathrm{MeV}$ and root-mean-square radius $0.92 \mathrm{fm}$ for the cutoff being $1.28 \mathrm{GeV}$. However, we can not make a definite conclusion because of the strong dependence of the results on the cutoff. The results of the state $\left(D^{(*)} \bar{B}^{(*)}\right)_{s}\left[J^{P}=1^{+}\right]$are similar to those of the state $\left(D^{(*)} \bar{B}^{(*)}\right)_{s}\left[J^{P}=0^{+}\right]$. Different from the $J^{P}=0^{+}, 1^{+}$case, one obtains a bound state of $\left(D^{(*)} \bar{B}^{(*)}\right)_{s}\left[J^{P}=2^{+}\right]$with binding energy $1.23 \mathrm{MeV}$ and root-meansquare radius $2.25 \mathrm{fm}$ for the cutoff being $2.20 \mathrm{GeV}$. The present meson-exchange model suggests that the $\left(D^{(*)} \bar{B}^{(*)}\right)_{s}\left[J^{p}=2^{+}\right]$might be a molecular candidate. 


\section{The Systems with Strangeness 2}

For the systems with strangeness $S=2$, there are no long range pion-exchange, but mediate-range sigma and eta exchanges and short-range phi-exchange.

\section{1. $\left(D^{(*)} D^{(*)}\right)_{s s}$ and $\left(\bar{B}^{(*)} \bar{B}^{(*)}\right)_{s s}$}

In the charmed sector, we obtain no bound state of $J^{P}=0^{+}$for the cutoff being less than $2.50 \mathrm{GeV}$. Differently, if we fix the cutoff to be $1.90 \sim 1.96 \mathrm{GeV}$, we obtain a loosely bound state of $\left(\bar{B}^{(*)} \bar{B}^{(*)}\right)_{s s}\left[J^{P}=0^{+}\right]$for which the dominant channel is $\bar{B}_{s} \bar{B}_{s}\left({ }^{1} S_{0}\right)$, with a probability around $90 \%$. Based on our results, $\left(\bar{B}^{(*)} \bar{B}^{(*)}\right)_{s s}$ might be a molecular candidate whereas $\left(D^{(*)} D^{(*)}\right)_{s s}$ is not. The results of the $J^{P}=1^{+}, 2^{+}$cases are very similar to those of the $J^{P}=0^{+}$case.

\section{2. $\left(D^{(*)} \bar{B}^{(*)}\right)_{s s}$}

The results of the three states $\left(D^{(*)} \bar{B}^{(*)}\right)_{s s}\left[J^{P}=0^{+}, 1^{+}, 2^{+}\right]$are very similar to each other. One can obtain loosely bound states with the cutoff being around 2.35 $\mathrm{GeV}$. Our numerical results suggest that all of the three states might be molecular candidates, but not ideal ones since the results depend a little sensitively on the cutoff.

Table 1. Summary of our results. "***" means the corresponding state is forbidden due to symmetry. " $\sqrt{ }$ " ("X") means the corresponding state might (not) be a molecule. " $\chi$ " denotes that the corresponding state is supported by not strongly to be a molecular candidate because the results depend a little sensitively on the cutoff. And, "?" means that we can not draw a definite conclusion with the present meson-exchange approach

\begin{tabular}{|c|c|c|c|c|c|c|c|c|}
\hline \multicolumn{3}{|c|}{ Double Charm } & \multicolumn{3}{|c|}{ Double Bottom } & \multicolumn{3}{|c|}{ Charm \& Bottom } \\
\hline$D^{(*)} D^{(*)}$ & $\begin{array}{l}0\left(0^{+}\right) \\
0\left(1^{+}\right) \\
0\left(2^{+}\right) \\
1\left(0^{+}\right) \\
1\left(1^{+}\right) \\
1\left(2^{+}\right)\end{array}$ & $\begin{array}{c}* * * \\
\sqrt{ } \\
\times \\
\times \\
\times \\
\times\end{array}$ & $\bar{B}^{(*)} \bar{B}^{(*)}$ & $\begin{array}{l}0\left(0^{+}\right) \\
0\left(1^{+}\right) \\
0\left(2^{+}\right) \\
1\left(0^{+}\right) \\
1\left(1^{+}\right) \\
1\left(2^{+}\right)\end{array}$ & $\begin{array}{c}* * * \\
\sqrt{ } \\
? \\
\not \\
\sqrt{ } \\
\not\end{array}$ & $D^{(*)} \bar{B}^{(*)}$ & $\begin{array}{l}0\left(0^{+}\right) \\
0\left(1^{+}\right) \\
0\left(2^{+}\right) \\
1\left(0^{+}\right) \\
1\left(1^{+}\right) \\
1\left(2^{+}\right)\end{array}$ & $\begin{array}{l}\not \\
\sqrt{ } \\
\sqrt{ } \\
\not \\
x \\
x\end{array}$ \\
\hline$\left(D^{(*)} D^{(*)}\right)_{s}$ & $\begin{array}{l}\frac{1}{2}\left(0^{+}\right) \\
\frac{1}{2}\left(1^{+}\right) \\
\frac{1}{2}\left(2^{+}\right)\end{array}$ & $\begin{array}{l}x \\
x \\
x\end{array}$ & $\left(\bar{B}^{(*)} \bar{B}^{(*)}\right)_{s}$ & $\begin{array}{l}\frac{1}{2}\left(0^{+}\right) \\
\frac{1}{2}\left(1^{+}\right) \\
\frac{1}{2}\left(2^{+}\right)\end{array}$ & $\begin{array}{l}\searrow \\
\sqrt{ } \\
\sqrt{ }\end{array}$ & $\left(D^{(*)} \bar{B}^{(*)}\right)_{s}$ & $\begin{array}{l}\frac{1}{2}\left(0^{+}\right) \\
\frac{1}{2}\left(1^{+}\right) \\
\frac{1}{2}\left(2^{+}\right)\end{array}$ & $\begin{array}{l}? \\
? \\
\not\end{array}$ \\
\hline$\left(D^{(*)} D^{(*)}\right)_{s s}$ & $\begin{array}{l}0\left(0^{+}\right) \\
0\left(1^{+}\right) \\
0\left(2^{+}\right)\end{array}$ & $\begin{array}{l}\times \\
\times \\
\times\end{array}$ & $\left(\bar{B}^{(*)} \bar{B}^{(*)}\right)_{s s}$ & $\begin{array}{l}0\left(0^{+}\right) \\
0\left(1^{+}\right) \\
0\left(2^{+}\right)\end{array}$ & $\begin{array}{l}\downarrow \\
\sqrt{ } \\
\sqrt{ }\end{array}$ & $\left(D^{(*)} \bar{B}^{(*)}\right)_{s s}$ & $\begin{array}{l}0\left(0^{+}\right) \\
0\left(1^{+}\right) \\
0\left(2^{+}\right)\end{array}$ & $\begin{array}{l}x \\
x \\
x\end{array}$ \\
\hline
\end{tabular}




\section{Conclusion}

In the present paper, we investigate the possible molecular states with two heavy quarks, including $D^{(*)} D^{(*)}$ with double charms, $\bar{B}^{(*)} \bar{B}^{(*)}$ with double bottoms and $D^{(*)} \bar{B}^{(*)}$ with both one charm and one bottom. In our study, we take into account the S-D mixing due to the tensor force arising from the pseudoscalar meson exchange and the vector meson exchange, and particularly, the coupled-channel effect in the flavor space.

Interestingly, we obtain some loosely bound states which are suggested to be searched for in the future experiment. And we summarize our results in Table 1.

\section{Acknowledgments}

This project was supported by the National Natural Science Foundation of China under Grants 11075004, 11021092, 11261130311, 11222547, 11175073, 11035006, Ministry of Science and Technology of China (2009CB825200), the Ministry of Education of China (FANEDD under Grant No. 200924, DPFIHE under Grant No. 20090211120029, NCET, the Fundamental Research Funds for the Central Universities) and the Fok Ying-Tong Education Foundation (No. 131006). This work is also supported in part by the DFG and the NSFC through funds provided to the sinogermen CRC 110 "Symmetries and the Emergence of Structure in QCD".

\section{References}

1. N. A. Tornqvist, (2003), arXiv:hep-ph/0308277 [hep-ph].

2. F. E. Close and P. R. Page, Phys. Lett. B578, 119 (2004).

3. E. S. Swanson, Phys. Lett. B588, 189 (2004).

4. N. A. Tornqvist, Phys. Lett. B590, 209 (2004).

5. S.-L. Zhu, Phys. Lett. B625, 212 (2005).

6. M. T. AlFiky, F. Gabbiani, and A. A. Petrov, Phys. Lett. B640, 238 (2006).

7. Y.-R. Liu, X. Liu, W.-Z. Deng, and S.-L. Zhu, Eur. Phys. J. C56, 63 (2008).

8. X. Liu, Y.-R. Liu, W.-Z. Deng, and S.-L. Zhu, Phys. Rev. D77, 034003 (2008).

9. X. Liu, Y.-R. Liu, W.-Z. Deng, and S.-L. Zhu, Phys. Rev. D77, 094015 (2008).

10. C. E. Thomas and F. E. Close, Phys. Rev. D78, 034007 (2008).

11. F. Close and C. Downum, Phys. Rev. Lett. 102, 242003 (2009).

12. G.-J. Ding, Phys. Rev. D79, 014001 (2009).

13. G.-J. Ding, J.-F. Liu, and M.-L. Yan, Phys. Rev. D79, 054005 (2009).

14. X. Liu, Z.-G. Luo, Y.-R. Liu, and S.-L. Zhu, Eur. Phys. J. C61, 411 (2009).

15. X. Liu and S.-L. Zhu, Phys. Rev. D80, 017502 (2009).

16. Y.-R. Liu and Z.-Y. Zhang, Phys. Rev. C79, 035206 (2009).

17. S. Ohkoda, Y. Yamaguchi, S. Yasui, K. Sudoh, and A. Hosaka, Phys. Rev. D86, 014004 (2012).

18. S. Ohkoda, Y. Yamaguchi, S. Yasui, K. Sudoh, and A. Hosaka, (2012), arXiv:1209.0144 [hep-ph].

19. F. Aceti, R. Molina, and E. Oset, PoS QNP2012, 072 (2012).

20. M. B. Voloshin and L. B. Okun, JETP Lett. 23, 333 (1976).

21. A. De Rujula, H. Georgi, and S. L. Glashow, Phys. Rev. Lett. 38, 317 (1977).

22. N. A. Tornquist, Nuovo Cim. A107, 2471 (1994). 
23. N. A. Tornquist, Z. Phys. C61, 525 (1994).

24. M. Mattson et al., SELEX Collaboration, Phys. Rev. Lett. 89, 112001 (2002).

25. A. Ocherashvili et al., SELEX Collaboration, Phys. Lett. B628, 18 (2005).

26. B. Aubert et al., BABAR Collaboration, Phys. Rev. D74, 011103 (2006).

27. R. Chistov et al., BELLE Collaboration, Phys. Rev. Lett. 97, 162001 (2006).

28. I. W. Lee, A. Faessler, T. Gutsche, and V. E. Lyubovitskij, Phys. Rev. D80, 094005 (2009).

29. N. Li and S.-L. Zhu, Phys. Rev. D86, 074022 (2012).

30. W. Meguro, Y.-R. Liu, and M. Oka, Phys. Lett. B704, 547 (2011).

31. N. Li and S.-L. Zhu, Phys. Rev. D86, 014020 (2012). 